\title{
MR
}

\section{Population genetic structure of critically endangered salamander (Hynobius amjiensis) in China: recommendations for conservation}

\author{
J. Yang ${ }^{1}$, C.S. Chen ${ }^{1,2}$, S.H. Chen ${ }^{1}$, P. Ding ${ }^{3}$, Z.Y. Fan ${ }^{1}$, Y.W. Lu ${ }^{1}$, L.P. Yu ${ }^{4}$ \\ and H.D. Lin \\ ${ }^{1}$ Zhejiang Museum of Natural History, Hangzhou, Zhejiang, China \\ ${ }^{2}$ College of Agriculture and Biotechnology, Zhejiang University, \\ Hangzhou, Zhejiang, China \\ ${ }^{3}$ The Key Laboratory of Conservation Biology for Endangered Wildlife of \\ the Ministry of Education, College of Life Sciences, Zhejiang University, \\ Hangzhou, China \\ ${ }^{4}$ The Administration Bureau of Longwangshan Natural Reserve, \\ Anji, Zhejiang, China \\ ${ }^{5}$ The Affiliated School of National Tainan First Senior High School, \\ Tainan, Taiwan \\ Corresponding authors: C.S. Chen / S.H. Chen \\ E-mail: ccs531@126.com / chensh@zmnh.com \\ Genet. Mol. Res. 15 (2): gmr.15027733 \\ Received September 25, 2015 \\ Accepted January 15, 2016 \\ Published June 10, 2016 \\ DOI http://dx.doi.org/10.4238/gmr.15027733
}

\begin{abstract}
Amji's salamander (Hynobius amjiensis) is a critically endangered species (IUCN Red List), which is endemic to mainland China. In the present study, five haplotypes were genotyped for the mtDNA cyt $b$ gene in 45 specimens from three populations. Relatively low levels of haplotype diversity $(\mathrm{h}=0.524)$ and nucleotide diversity $(\pi=0.00532)$ were detected. Analyses of the phylogenic structure of $H$. amjiensis showed no evidence of major geographic partitions or substantial barriers to historical gene flow throughout the species' range. Two major phylogenetic haplotype groups were revealed, and were
\end{abstract}


estimated to have diverged about 1.262 million years ago. Mismatch distribution analysis, neutrality tests, and Bayesian skyline plots revealed no evidence of dramatic changes in the effective population size. According to the SAMOVA and STRUCTURE analyses, $H$. amjiensis should be regarded as two different management units.

Key words: Hynobius amjiensis; Mitochondrial DNA; Management units; Conservation

\section{INTRODUCTION}

A fundamental tenet of conservation biology is to identify and protect the evolutionary heritage and future evolutionary potential of threatened species (Monsen and Blouin, 2003). Phylogeographic analysis has become a major tool for investigating historical aspects of biogeography and population genetic structure. An understanding of the evolutionary history and genetic structure of a species is of critical importance when designing conservation strategies. Molecular studies in amphibians have commonly revealed strong phylogeographic structure and limited gene flow among populations, and have shown high levels of intraspecific mitochondrial divergence (Vieites et al., 2006). In recent decades, molecular markers have been used extensively to study phylogeographical patterns in different amphibians, and to group populations of species into distinct units for conservation in mainland China (Murphy et al., 2000; Zhang et al., 2008; Zhang et al., 2010). Conservation management authorities are increasingly recognising the importance of protecting genetic diversity within a species.

Amji's salamander (Hynobius amjiensis) is a critically endangered species (IUCN Red List) that is endemic to mainland China. This species is aquatic and currently only found on Qianmutian of Longwangshan, Zhejiang province (the type locality), and two sites on Qingliangfeng between Anhui and Zhejiang province (Fu et al., 2003; Li et al., 2013) (Figure 1). The China State Forestry Administration listed H. amjiensis as an extremely threatened species in 2012's National Biodiversity Survey, and the IUCN Red List considers H. amjiensis as critically endangered (Gu and Lau, 2004; Wang and Xie, 2004). Few basic studies have

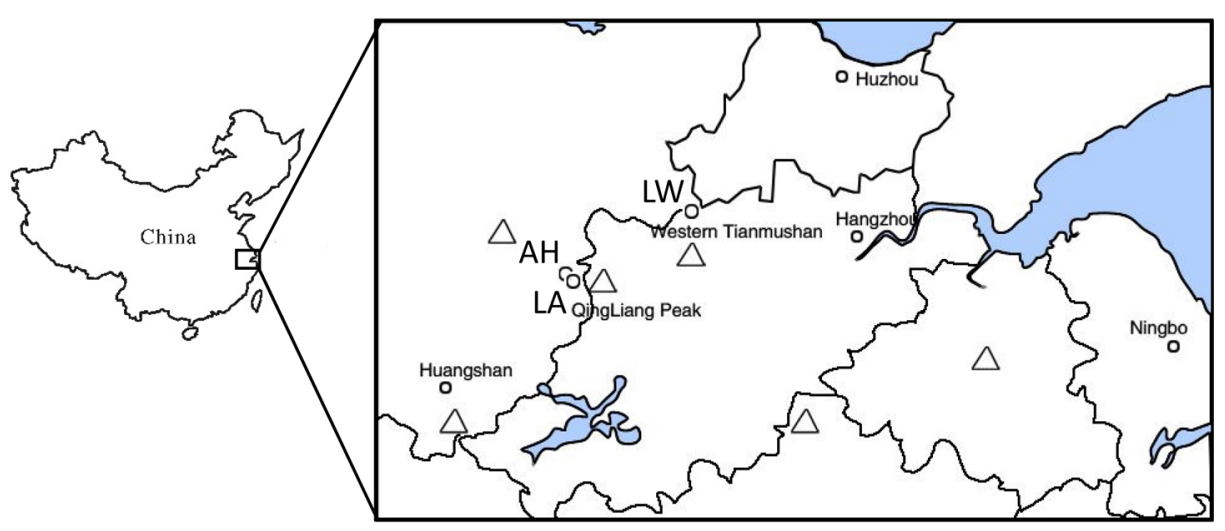

Figure 1. Map showing the three sampling sites of Hynobius amjiensis in mainland China. Collection sites (circles) correspond to locations defined in the text and in Table 1. 
been performed on the breeding characteristics of H. amjiensis (Gu et al., 1999) and ecological factors of its microhabitat (Ye, 2012); however, the extent of genetic structure and divergence among $H$. amjiensis populations remains unknown.

Documenting and maintaining the genetic diversity of populations are central issues in conservation biology (Dufresnes et al., 2013). From a conservation perspective, two types of independent units within a population of a species are suggested (Moritz, 1994). A previous study by our group provided a means of assessing the evolutionary distinctiveness of $H$. amjiensis populations that may be in need conservation. Those data can be used to establish management units (MUs) and/or evolutionary significant units (ESUs), which are two commonly used designations for threatened or endangered taxa (Moritz, 1994). ESUs are designated based on reciprocal monophyly at mitochondrial markers, whereas MUs are identified by significant differences in allele frequency distribution and significant divergence in mitochondrial or nuclear loci (Moritz, 1994). Analysis of mitochondrial DNA (mtDNA) is a powerful tool for detecting population genetic structure at the intraspecific level (Avise et al., 1987), due primarily to its maternal mode of inheritance, lack of recombination, high mutation rate, and the process of lineage extinction within populations (Waits et al., 1998). Reduced genetic diversity as a result of drift (bottleneck) is exacerbated in mitochondrial genes because they only possess a quarter of the effective population size of unclear genes (Nei, 1983). In the present study, the mtDNA cytochrome $b$ gene was used to assess genetic variation within and among populations of $H$. amjiensis in mainland China. The objectives of our study were 1) to identify the phylogenetic and geographic relationships among these populations, 2) to propose historical biogeographic and demographic scenarios to accommodate the genetic variation observed among and within lineages, 3) to raise issues regarding the practice of designating ESUs and MUs, and 4) to assess whether the current management strategies for this species are sufficient to maintain its long-term survival.

\section{MATERIAL AND METHODS}

\section{Sampling and molecular analyses}

We collected three populations of $H$. amjiensis across its entire distribution range in China between 2009 and 2014. Sampling sites were located on Qianmutian of Longwangshan (LW), Qingliangfeng (LA) in Zhejiang province, and Anhui (AH) in Anhui province. Information on the geographic locations sampled in this study is shown in Table 1 and Figure 1. All individuals were adult frogs from which a piece of tail tip was obtained. Samples were preserved in $95 \%$ ethanol or frozen for subsequent DNA extraction. Total genomic DNA was extracted from tail tips using a DNAeasy tissue extraction kit (Qiagen) following the manufacturer instructions, or using traditional proteinase $\mathrm{K}$ digestion at $37^{\circ}$ or $55^{\circ} \mathrm{C}$ and phenol-chloroform extraction protocols.

PCR was carried out using the primers HAcytb-F (5'-ACCGAAACTTCTGGCTTGA A-3') and HAcytb-R (5'-GGCTTACAAGACCGATGCTT-3') for the cyt $b$ fragment, which were designed based on the complete mitochondrial sequence region of $H$. amjiensis deposited in NCBI (DQ333808). Each 100- $\mu \mathrm{L}$ PCR reaction contained $10 \mathrm{ng}$ template DNA, $10 \mu \mathrm{L} 10 \mathrm{X}$ reaction buffer, $10 \mu \mathrm{L}$ dNTP mix $(8 \mathrm{mM}), 10$ pmol each primer, and $4 \mathrm{U}$ Taq polymerase (Promega, Madison, WI, USA). PCR was conducted in a thermal cycler (Eppendorf Mastercycler) using the following conditions: one cycle of denaturation at $95^{\circ} \mathrm{C}$ for $4 \mathrm{~min}, 30$ cycles of de- 
naturation at $94^{\circ} \mathrm{C}$ for $45 \mathrm{~s}$, annealing at $48^{\circ} \mathrm{C}$ for $1 \min 15 \mathrm{~s}$, and extension at $72^{\circ} \mathrm{C}$ for $1 \mathrm{~min}$ $30 \mathrm{~s}$, followed by $72^{\circ} \mathrm{C}$ extension for $10 \mathrm{~min}$ and $4^{\circ} \mathrm{C}$ for storage. PCR products were purified by electrophoresis in a 1.0\% agarose gel using $1 \mathrm{X}$ TAE buffer. The gel was stained with ethidium bromide and the desired DNA band was cut and eluted using an Agarose Gel Purification Kit (QIAGEN, Valencia, CA, USA). PCR products were then subjected to cycle sequencing reactions conducted by Sangon Biotec (Shanghai) Co., Ltd using an ABI PRISM 3730XL sequencer with the BigDye Terminator kit (Applied Biosystems). All sequences have been deposited in GenBank under the following inclusive accession No. KU557696-KU557740.

Table 1. List of sampling locations and latitudes and longitudes, sample sizes, haplotype numbers, haplotype diversities $(h)$, nucleotide diversities $(\pi)$, Tajima's $D$ and Fu's $(F s)$ test, and Ramos-Onsins and Rozas' $R_{2}$ of mtDNA cyt $b$ sequences for each location.

\begin{tabular}{|c|c|c|c|c|c|c|c|}
\hline Locations (abbreviation) & \begin{tabular}{|l|} 
Latitude/longitude \\
\end{tabular} & Sample size & Haplotype numbers & \begin{tabular}{|l} 
Haplotype diversity $(h)$ \\
\end{tabular} & \begin{tabular}{|l} 
Nucleotide diversity $(\pi)$ \\
\end{tabular} & Tajima's $D$ & \begin{tabular}{c|r} 
Fu's $F_{S}$ & $R_{2}$ \\
-0.606 & 0.1496
\end{tabular} \\
\hline shan (LW) & 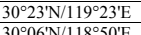 & $\frac{20}{5}$ & 4 & 0.432 & 0.00067 & -1.73887 & \begin{tabular}{c|c|}
-0.606 & 0.1496 \\
0.626 & 0.3000 \\
\end{tabular} \\
\hline $\begin{array}{l}\text { Qinmourliangfeng (LA) } \\
\text { Qing }\end{array}$ & $30^{\circ} 05^{5} \mathrm{~N} / 118^{\circ} 51^{\circ} \mathrm{E}$ & $\frac{5}{20}$ & $\frac{2}{3}$ & 0.000 & & $\frac{1.22474}{2.44469}$ & \begin{tabular}{|c|c|}
0.326 & 0.3000 \\
13.363 & 0.2288 \\
\end{tabular} \\
\hline Total & & 45 & 5 & 0.524 & 0.00532 & 0.20661 & 0.1195 \\
\hline
\end{tabular}

\section{Genetic diversity, phylogenetic, and phylogeographic analysis}

All nucleotide sequences of the cyt $b$ sequences were aligned using Clustal X v1.81 (Thompson et al., 1997) with final minor edits completed manually. We calculated the nucleotide diversity $(\pi)$, number of haplotypes $(\mathrm{N})$, and haplotype diversity $(h)$ for each population using the software DnaSP 5.0 (Librado and Rozas, 2009). Our molecular phylogenetic tree for $H$. amjiensis was separately reconstructed by neighbor-joining (NJ) tree and Bayesian analysis using MEGA v6.0 (Tamura et al., 2013) and MrBayes v3.2 (Ronquist et al., 2012), respectively. NJ tree nodes and branch lengths were statistically tested using a bootstrap method of 10,000 replicates and an interior branch test, respectively. The ModelTest program input into PhyML v. 3.0 (Guindon et al., 2010) was used to determine the best fitting models chosen using the Akaike Information Criterion (AIC). Markov Chain Monte Carlo simulations were run for $5,000,000$ generations with trees sampled every 1000 generations. Next, Bayesian posterior probabilities were estimated after omitting the initial 1,000,000 generations. We sampled a tree every 100 generations and calculated a consensus topology for 7500 trees by omitting the first 2500 trees. Additionally, Median-Joining (MJ) networks were constructed with the program Network 4.6.1.0. software (http://www.fluxus-engineering.com/).

The presence of phylogeographic structure was estimated by measures of differentiation: $G_{\mathrm{ST}}$, which takes only haplotype frequencies into account, and $N_{\mathrm{ST}}$, which considers similarities between haplotypes in addition to their frequencies (Pons and Petit, 1996). A greater $N_{\mathrm{ST}}$ value means that more closely related haplotypes occur in the same population, indicating phylogeographic structure (Pons and Petit, 1996), $G_{\mathrm{ST}}$ and $N_{\mathrm{ST}}$ were calculated using DnaSP v5.0 (Librado and Rozas, 2009). Differences between populations were assessed with pairwise genetic differentiation values $\left(F_{\mathrm{ST}}\right)$ and hierarchical analysis of molecular variance (AMOVA) in Arlequin v3.5 (Excoffier and Lischer, 2010), using the following groups according to the geographic barriers: two independent groups including the Longwangshan group (LW) and the Qingliangfeng group (LA and AH), which were primarily divided by the West Tianmushan mountain range. To define geographically homogenous groups of populations, we performed a spatial analysis of molecular variance using SAMOVA 
v1.0 (Dupanloup et al., 2002). The program partitions the populations into a specified number of groups with the greatest variation among groups $\left(F_{\mathrm{CT}}\right)$ (Dupanloup et al., 2002). Geographic distances among populations were estimated based on their coordinates using Google Earth. Correlations between the pairwise geographical and genetic distances were estimated using Mantel tests implemented in Arlequin v3.5 (Excoffier and Lischer, 2010).

To infer the population demographic history of $H$. amjiensis, several methods were used including, Tajima's $D$ test, Ramos-Onsins and Rozas' $R_{2}$ and Fu's $F_{S}$-test of neutrality in the software DnaSP v 5.0 (Librado and Rozas, 2009). Historical demographic expansion events of each evolutionary lineage were examined using mismatch distribution, as implemented in Arlequin v3.5 (Excoffier and Lischer, 2010). A multimodal distribution of pairwise differences was displayed in populations at demographic equilibrium or in decline, whereas a unimodal distribution was displayed in populations that had experienced a sudden demographic expansion. The time to the most recent common ancestor (TMRCA) for major mitochondrial lineages under a MCMC (Markov Chain Monte Carlo) Bayesian approach was estimated with the software BEAST v1.7.5 (Drummond and Rambaut, 2007). These analyses estimated genealogies and divergence dates for all nodes and were sampled every 1000th iteration for 10,000,000 generations with $10 \%$ of the initial samples discarded as burn-in. Multiple calibrated salamander studies have found cyt $b$ substitution rates in the range of 0.64\%/MYR (million years) (Bayer et al., 2012). All analyses were performed using the HKY model of nucleotide substitution. The analysis was run five times to test for the stability and convergence of MCMC chains in plots of posterior log likelihoods in Tracer v1.6 (Rambaut et al., 2014). To identify the number of genetically distinct clusters (K) based only on the allele frequency data, in order to generate membership assignment coefficients for all individuals, a Bayesian admixture procedure was used Structure v2.3 (Pritchard et al., 2000). Sequence data were converted to Structure input file format using the program xmfa2struct (available at: http://www.xavierdidelot.xtreemhost.com/clonalframe.htm). The admixture ancestry model was used, which assumed correlated allele frequencies among populations. The number of subpopulations $(\mathrm{K})$ was estimated using 20 independent runs with $\mathrm{K}=1-3$ (assuming no prior population delineation information) at 1,000,000 MCMC repetitions combined with a 100,000 repetition burn-in period. We determined the most appropriate value of $\mathrm{K}$ for our dataset using the method of Evanno et al. (2005), as implemented in STRUCTURE HARVESTER (Earl, 2012). We selected the value of $K$ that best fitted our data using the log posterior probability of the data for a given K, Ln Pr (XjK) (Pritchard and Wen, 2004).

\section{RESULTS}

\section{Genetic diversity of $\mathrm{H}$. amjiensis in China}

After alignment, DNA sequences of the complete cyt $b$ gene were $1140 \mathrm{bp}$ in length. A total of five mtDNA haplotypes were identified, of which four were found in LW, two were found in $\mathrm{AH}$, and three in LA. Alignment of all cyt $b$ gene sequences revealed that 25 positions were polymorphic, of which 22 were parsimony informative. The average G, A, T, and C base composition was $13.8,29.3,29.9$, and $27.0 \%$, respectively. The average haplotype diversity (h) value was 0.524 , ranging from 0.432 (LW) to $0.600(\mathrm{AH})$ within populations (Table 1). The average nucleotide diversity $(\pi)$ value was 0.00532 , with the highest nucleotide diversity observed in LA ( $\pi=0.00890)$, and the lowest in AH $(\pi=0.00053)$ (Table 1$)$. 


\section{Phylogenetic reconstruction}

The phylogeographic structure was examined by contrasting two values representative of genetic differentiation: $G_{\mathrm{ST}}$ and $N_{\mathrm{ST}}$ (Pons and Petit, 1996). $N_{\mathrm{ST}}$ takes into account the number of differences among haplotypes, whereas $G_{\mathrm{ST}}$ only takes into account haplotype frequencies. A comparison of two derived fixation indices revealed a close relationship between phylogeny and geography, with $N_{\mathrm{ST}}$ being much larger than $G_{\mathrm{ST}}(0.286$ and 0.056 , respectively), revealing the presence of phylogeographic structure. The optimum model of substitution selected by jModelTest was HKY+I for the cyt $b$ gene. NJ and Bayesian phylogenetic analysis reconstructed using mtDNA cyt $b$ sequences (Figure 2) recovered two highly supported, geographically structured lineages in $H$. amjiensis (Figure 2). Two major phylogroups were identified: Clade A consists of three populations and Clade B only includes the LW population. The $H$. amjiensis cyt $b$ haplotype network comprised five haplotypes, haplotype H1 is the most frequent ( 30 individuals) and is found in individuals from all collection sites, followed by H5 (7 individuals) and H3 (6 individuals). Haplotype H5 is found in the LA population. Furthermore, haplotypes $\mathrm{H} 2$ and $\mathrm{H} 4$ are unique in the LW population (Figure 3).

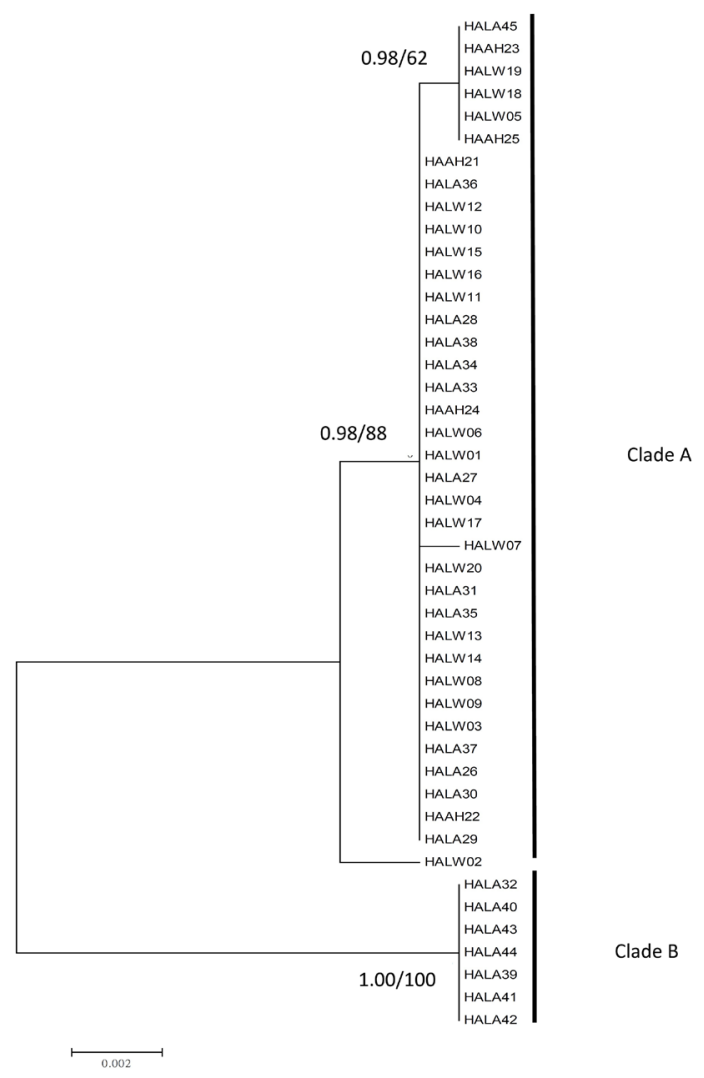

Figure 2. Neighbor-joining (NJ) tree of genetic relationships among three populations of Hynobius amjiensis using the mitochondrial cyt $b$ sequences obtained from 45 individuals. Values above branches represent the posterior probabilities for the Bayesian analysis and bootstrap values for the NJ analyses. 


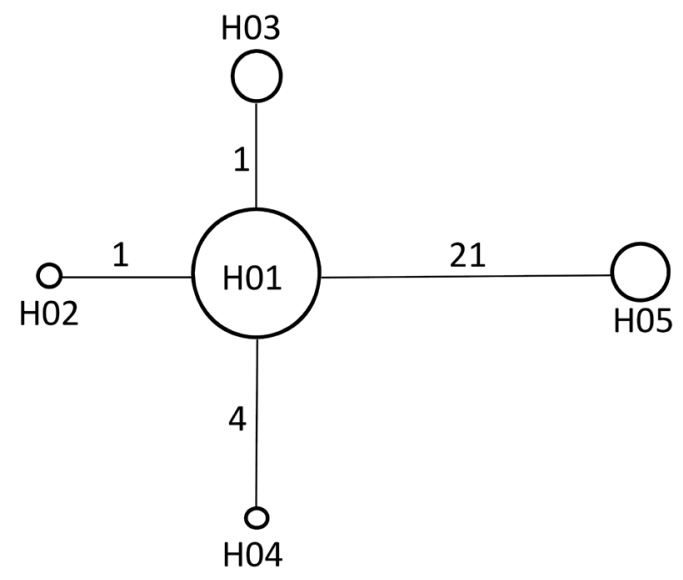

Figure 3. A minimum spanning network constructed using the five mitochondrial cyt $b$ haplotypes identified in Hynobius amjiensis. Haplotype designations (Table 1) are indicated next to each circle. Locality designations (see Figure 1) for specimens possessing each haplotype are indicated inside the circles. The sizes of the circles are proportional to the number of individuals represented. The length of the lines between circles is roughly proportional to the estimated number of mutational steps between the haplotypes.

\section{Demographic history}

An examination of demographic histories revealed marked differences among the clades and populations. Low haplotype diversity and low nucleotide diversity was detected in each population (Table 1). The results of Fu's Fs, Tajima's D-values, and the $\mathrm{R}_{2}$ test were not statistically significant for any of the populations. Furthermore, the overall mismatch distribution was clearly multimodal; therefore, a fit to the sudden expansion model of Rogers (Rogers, 1995) was significantly rejected (Figure 4). The Bayesian skyline plot revealed a long period of relatively constant population size and showed a recent decreasing trend in population size since c. 0.025 million years ago (mya) for $H$. amjiensis (Figure 5).

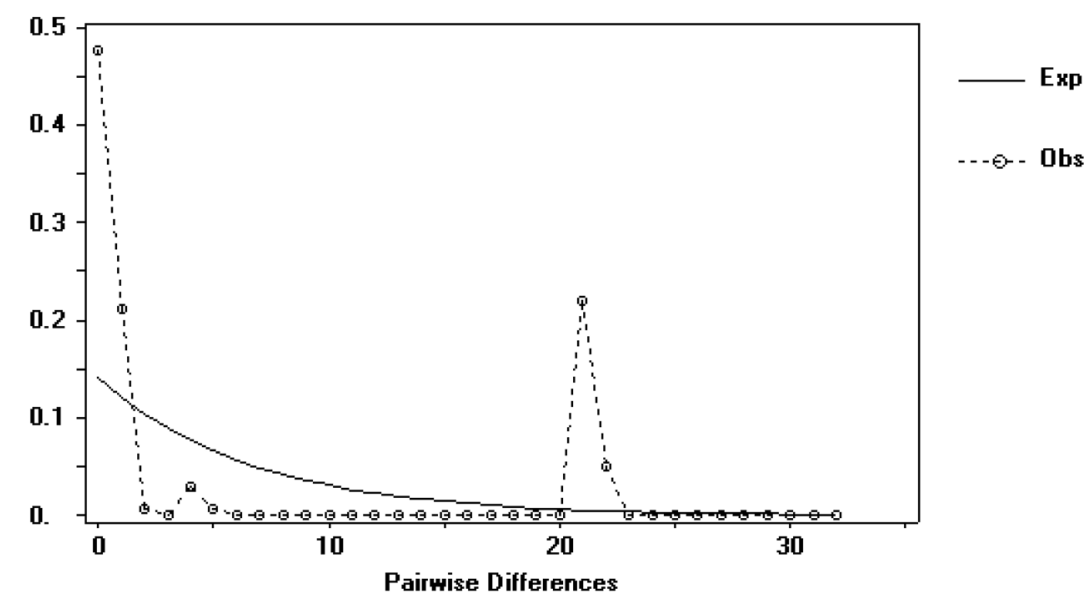

Figure 4. Mismatch distribution among haplotypes of Hynobius amjiensis. 


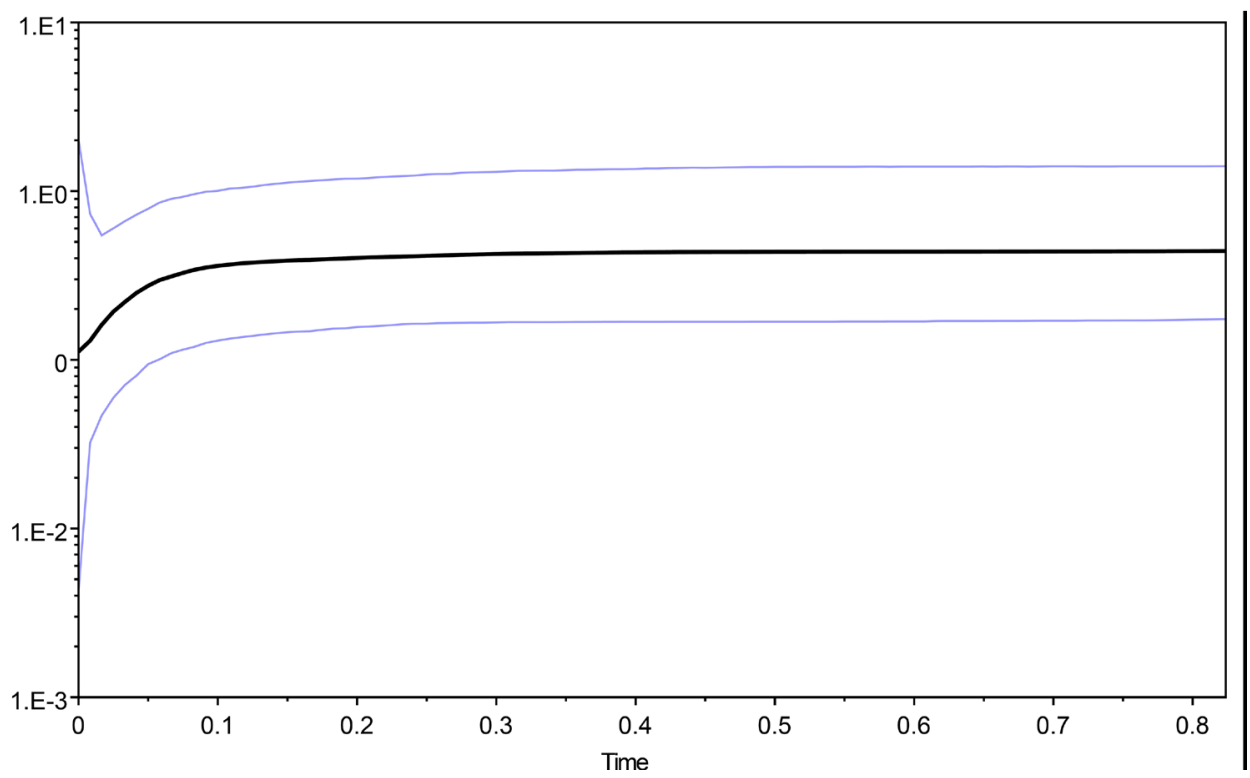

Figure 5. Bayesian skyline plots showing the demographic history of total populations of Hynobius amjiensis. Dark lines represent median values of the $\log _{10}$ of the population size, and blue lines represent the $95 \%$ highest probability density (HPD) intervals.

\section{Population genetic analysis}

The overall standardized $F_{\text {ST }}$ value among all samples was 0.286 and pairwise $F_{\text {ST }}$ values revealed genetic differentiation among three populations of $H$. amjiensis, with values ranging from -0.024 to 0.291 (Table 2). For the AMOVA hierarchical analysis, populations were grouped according to the zoogeographic zone $(\mathrm{N}=2)$. In the AMOVA, most of the molecular variance $(86.81 \%)$ was attributable to variation within populations, with $39.50 \%$ among populations within groups, and $-26.30 \%$ among lineages (Table 3). Spatial analysis of molecular variance (SAMOVA) indicated that the value of $F_{\text {СТ }}$ was highest when $\mathrm{K}=2$ $\left(F_{\mathrm{CT}}=0.384, \mathrm{P}=0.000\right)$, in which LA formed an unique group, and $\mathrm{AH}$ and $\mathrm{LW}$ formed another group. Most of the remaining variation was within populations $(69.12 \%)$ or among populations within groups $(-7.60 \%)$ (Table 3$)$. A Mantel test $(r=-0.000435, \mathrm{P}=0.68)$ did not detect a correlation between genetic distance and geographic distance. Non-significant results for isolation-by-distance indicate that the gene flow among populations of $H$. amjiensis is little congruence with geographic proximity of populations.

Analyses of mitochondrial data using the STRUCTURE clustering algorithm (Pritchard, et al., 2000) indicated the presence of two distinct genetic clusters $(\mathrm{K}=2)(\mathrm{Ln}$ $\mathrm{P}(\mathrm{K})=-52.04$; variance $\operatorname{Var}[\operatorname{Ln} \mathrm{P}(\mathrm{D})]=410.720 ; \Delta \mathrm{K}=212.515)$ according to the $\Delta \mathrm{K}$ metric developed by Evanno et al. (2005). Most individuals of populations LW and AH, and equal portions of individuals from LA formed one group. The remaining individuals of the LA population formed the other group (Figure 6). 
Table 2. Matrix of pairwise $F_{\mathrm{ST}}$ (below diagonal) and $P$ values (above diagonal) between three populations based on mtDNA. Referred to Table 1 for the abbreviations of localities.

\begin{tabular}{l|c|c|c}
\hline & LW & AH & LA \\
\hline LW & & 0.666 & 0.009 \\
\hline AH & -0.024 & & 0.135 \\
\hline LA & 0.291 & 0.161 & \\
\hline
\end{tabular}

Table 3. AMOVA and SAMOVA results for analysis of genetic subdivision between populations based on mtDNA among geographic districts.

\begin{tabular}{|c|c|c|c|c|}
\hline & Sum of squares & Percentage of variation & Fixation indices & Significance tests \\
\hline \multicolumn{5}{|l|}{ Groups: (AMOVA, $\mathrm{K}=2$, geography) } \\
\hline Among groups & 3.350 & -26.30 & $F_{C T}=-0.26302$ & $\mathrm{P}=1.00000$ \\
\hline Among populations within groups & 25.250 & 39.50 & $\mathrm{FSC}_{\mathrm{SC}}=0.31271$ & $\mathrm{P}=0.00880$ \\
\hline Within populations & 105.000 & 86.81 & $\mathrm{~F}_{\mathrm{ST}}=0.13194$ & $\mathrm{P}=0.00782$ \\
\hline \multicolumn{5}{|l|}{ Groups: (SAMOVA, $\mathrm{K}=2$ ) } \\
\hline Among groups & 28.300 & 38.48 & $\mathrm{~F}_{\mathrm{CT}}=0.38485$ & $\mathrm{P}=0.00000$ \\
\hline Among populations within groups & 0.300 & -7.60 & $F_{S C}=-0.12360$ & $\mathrm{P}=0.31574$ \\
\hline Within populations & 105.000 & 69.12 & $\mathrm{~F}_{\mathrm{ST}}=0.30882$ & $\mathrm{P}=0.00000$ \\
\hline
\end{tabular}

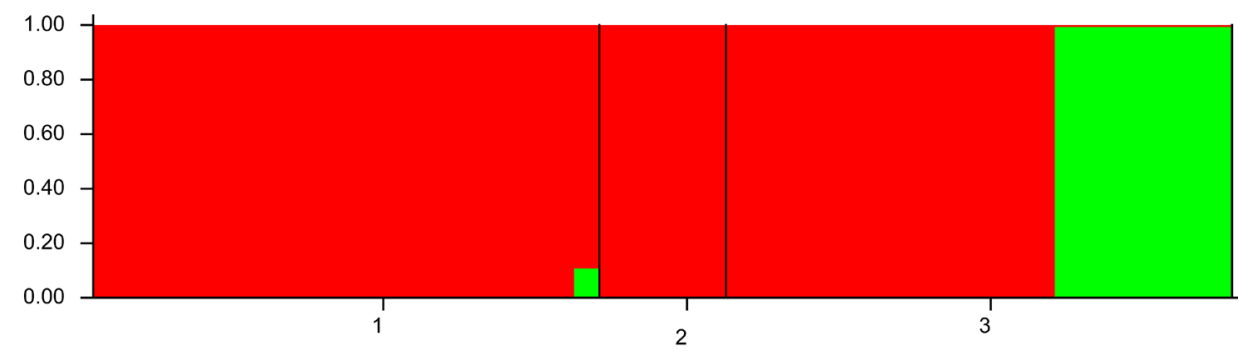

Figure 6. Clustering of individuals by Structure at $\mathrm{K}=2$. Individuals are represented by vertical bars, with each color representing one cluster and the length of the colored segment indicating the individual's estimated degree of kinship to that cluster (Y-axis). The different colors correspond to the population designations given at the bottom of the figure (X-axis). Populations are separated by black bars and are defined in Table 1.

\section{Molecular dating}

Based on the Bayesian coalescent method, the total population and clade A are estimated to have diverged from their common ancestor approximately $1.262(95 \% \mathrm{CI}=0.747$ $1.818)$ mya and $0.758(95 \% \mathrm{CI}=0.458-1.096)$ mya, respectively.

\section{DISCUSSION}

\section{Genetic diversity in Hynobius amjiensis}

Genetic diversity, in terms of haplotype diversity $(h)$ and nucleotide diversity $(\pi)$, was found to be lower in $H$. amjiensis compared to other salamanders [e.g., Hydromantes (Chiari, et al., 2012); Hynobius tokyoensis (Matsui, et al., 2007); Salamandrella keyserlingii, (Malyarchuk, et al., 2010)]. The loss of genetic diversity could be susceptible to stochastic processes within $H$. amjiensis if these populations have recently undergone a reduction in their effective population size. Considering that populations of $H$. amjiensis occur in very 
restricted geographical areas, genetic drift within these small demes may have resulted in reduced genetic variability, which is a common feature in endangered species (Frankham, 2005). The low densities of $H$. amjiensis at occupied sites and the current absence of $H$. amjiensis from potential breeding habitats throughout the historical range of this species may be contributing to increased fragmentation and isolation of populations (Chen and Yang, 2012). For H. amjiensis, our unpublished data indicate low local population densities of 65-90 individuals per hectare (Chen and Yang, 2012). We found higher levels of genetic diversity in LA populations, presumably due to the existence of high-quality habitat and the larger population size. The lowest genetic diversity was found in $\mathrm{AH}$, which is the population with the smallest sample size. The extinction of genetically volatile populations has resulted in the loss of a significant proportion of the species genetic diversity and this process of genetic erosion continues as small populations approach extinction.

\section{Phylogeography, population differentiation, and demographic changes in Hynobius amjiensis}

Landscape barriers may have influenced animal migrations between areas of mainland China during glaciations, thus shaping the genetic structure. However, analyses of the phylogeny structure of $H$. amjiensis revealed no evidence of major geographic partitions or substantial barriers to historical gene flow throughout the species' range. However, two alternative historic scenarios might result in polyphyly: (i) a recent separation of the two populations leading to incomplete lineage sorting, or (ii) a secondary admixture following range expansion (Avise, 2000). The haplotype network revealed a genetically homogeneous group of populations within $H$. amjiensis, with a common haplotype related to five haplotypes that differ by only one or four substitutions, with the exception of haplotype H5. In the present study, this pattern is likely to be a consequence of the low mitochondrial mutation rate found in turtles rather than a consequence of limited sample size.

A high degree of population genetic structure $\left(\mathrm{F}_{S T}=0.286\right)$ was observed in $H$. amjiensis, and we suggest that isolation is an important mechanism for differentiation among these populations. Tests of phylogeographical structure indicated significant structuring of mtDNA diversity within $H$. amjiensis, but AMOVA revealed that groupings of populations by geographic zone explained relatively little of the partitioning of genetic diversity within the subsection. These results indicate that the Longwangshan has not acted as an evident topographical barrier for $H$. amjiensis. However, according to the pairwise $\mathrm{F}_{S T}$ population differentiation analyses, two population groups of $H$. amjiensis occur, one on LW and AH, and the other on LA. According to the results of SAMOVA, two groups were obtained that revealed the highest percentage of differentiation among groups $(38.48 \%)$ and the smallest percentage of variation among populations within groups $(-7.60 \%)$. This pattern was well supported by the results of the STRUCTURE analysis, in which these two groups showed different patterns under $\mathrm{K}=2$. In general, population differentiation in salamander may also be due to poor dispersal ability. For $H$. amjiensis, our unpublished data indicate low local population sizes, although nothing is currently known about individual dispersal distances. However, it is also known that the main environmental factors influencing locomotion capacity are low temperatures and habitats with low vegetation cover (Ye, 2012). Haplotype H5 is only found in the LA population and differs from other haplotypes by 21 substitutions. With little or no ongoing gene flow, differences in haplotype frequency, the proportion of private alleles, and 
allelic sequence divergences should increase among populations with time (Ishida and Taylor, 2007). Our results suggested that incomplete lineage sorting from an ancestral polymorphic gene pool could be the cause of the mitochondrial polyphyly detected in $H$. amjiensis.

Nonsignificant values of Tajima's D and Fu's Fs statistics, and other demographic analyses (mismatch analysis and Bayesian skyline plots) also reveal no evidence of dramatic changes in the effective population size over the last several thousand years. Bayesian skyline plot analysis provided apparent evidence of a reduction in the effective population size and strongly indicated that the population has been demographically stable over the last 25,000 years. H. amjiensis prefers oviposition sites in small, cool, and weak acidic pools at high elevations (1300-1600 m above sea level). The warming climate presumably caused $H$. amjiensis to move from low to high elevations during the last glacial maximum (LGM, 2325,000 years ago) retreat, leading to a major reduction in suitable ecological zones during the post-glacial period, and dramatic reductions in their population size. Moreover, no evidence of an isolation-by-distance pattern implies a balance between drift and gene flow, due to ancestral allelic variation. Environmental shifts in montane regions, induced by climatic changes during the Pleistocene glacial cycles, have resulted in population divergence on different mountains (Hewitt, 1996). The MRCA of total populations and clade A existed approximately 1.262 mya and 0.758 mya, respectively. These two dates are consistent with the early Pleistocene. After an early split, subsequent fragmentation probably arose following the successive cycles of cooling and warming climatic conditions that characterize the Pleistocene (Zagwijn, 1992).

\section{Implications for conservation}

The goals of modern conservation biology are not only to preserve species and habitats, but also to conserve their evolutionary potential in terms of maintaining the genetic diversity of the extant species. Maintenance of long term processes that aid species evolution are a challenge for conservation managers. For the effective conservation and management of biological resources, assigning ESUs and MUs is very important (Moritz, 1994). Our data provide no support for the existence of distinct phylogeographic breaks within this species' range, implying that there are no historically isolated groups that should be viewed as separate ESUs (Moritz, 1994). For the different MUs, we suggest that protection is required synchronously because of their genetic uniqueness. We suggest that two groups identified by SAMOVA should be regarded as two different MUs in H. amjiensis. Narrowly distributed species are at a greater risk of extinction than more widely distributed taxa because catastrophic environmental change may simultaneously affect all individuals of a spatially restricted species (Riberon, et al., 2002). However, our results argue for the management of this species as a single population, given the ecological exchangeability of extant stocks, and support the ongoing reintroduction of three mixed extant populations.

In conclusion, the results of our study show that $H$. amjiensis has a low level of genetic structure among geographical populations. Populations of $H$. amjiensis occur in very restricted geographical areas, and genetic drift within these small demes may have resulted in reduced genetic variability, which is a common feature in endangered species The Bayesian skyline plot revealed a long period of relatively constant population size and showed a recent decreasing trend in population size since c. 0.025 mya for $H$. amjiensis. However, our results argue for the management of this species as a single population, given the ecological exchangeability of extant stocks, and support the ongoing reintroduction of three mixed extant populations. 


\section{Conflicts of interest}

The authors declare no conflict of interest.

\section{ACKNOWLEDGMENTS}

We thank Shuyan Zhang and Rui Guo from Zhejiang Qingliangfeng National Nature Reserve Administration for their field assistance. We are grateful for support from the Longwangshan Nature Reserve Administration and the Zhejiang Qingliangfeng National Nature Reserve Administration. This work was funded by the Zhejiang Provincial Natural Science Foundation (\#LQ12C06001).

\section{REFERENCES}

Avise JC (2000). Phylogeography: the history and formation of species. Harvard University Press.

Avise JC, Arnold J, Ball RM, Bermingham E, et al. (1987). Intraspecific phylogeography: the mitochondrial DNA bridge between population genetics and systematics. Annu. Rev. Ecol. Evol. Syst. 18: 489-522. http://dx.doi.org/10.1146/ annurev.es.18.110187.002421

Bayer CS, Sackman AM, Bezold K, Cabe PR, et al. (2012). Conservation genetics of an endemic mountaintop salamander with an extremely limited range. Conserv. Genet. 13: 443-454. http://dx.doi.org/10.1007/s10592-011-0297-7

Chen CS and Yang J (2012). Habitat monitoring of Hynobius amjiensis in Longwangshan, China. National Wetland 3.

Chiari Y, van der Meijden A, Mucedda M, Lourenço JM, et al. (2012). Phylogeography of Sardinian cave salamanders (genus Hydromantes) is mainly determined by geomorphology. PLoS One 7: e32332. http://dx.doi.org/10.1371/ journal.pone. 0032332

Drummond AJ and Rambaut A (2007). BEAST: Bayesian evolutionary analysis by sampling trees. BMC Evol. Biol. 7: 214. http://dx.doi.org/10.1186/1471-2148-7-214

Dufresnes C, Wassef J, Ghali K, Brelsford A, et al. (2013). Conservation phylogeography: does historical diversity contribute to regional vulnerability in European tree frogs (Hyla arborea)? Mol. Ecol. 22: 5669-5684. http://dx.doi. org $/ 10.1111 / \mathrm{mec} .12513$

Dupanloup I, Schneider S and Excoffier L (2002). A simulated annealing approach to define the genetic structure of populations. Mol. Ecol. 11: 2571-2581. http://dx.doi.org/10.1046/j.1365-294X.2002.01650.x

Earl DA (2012). STRUCTURE HARVESTER: a website and program for visualizing STRUCTURE output and implementing the Evanno method. Conserv. Genet. Resour. 4: 359-361. http://dx.doi.org/10.1007/s12686-011-9548-7

Evanno G, Regnaut S and Goudet J (2005). Detecting the number of clusters of individuals using the software STRUCTURE: a simulation study. Mol. Ecol. 14: 2611-2620. http://dx.doi.org/10.1111/j.1365-294X.2005.02553.x

Excoffier L and Lischer HE (2010). Arlequin suite ver 3.5: a new series of programs to perform population genetics analyses under Linux and Windows. Mol. Ecol. Resour. 10: 564-567. http://dx.doi.org/10.1111/j.1755-0998.2010.02847.x

Frankham R (2005). Genetics and extinction. Biol. Conserv. 126: 131-140. http://dx.doi.org/10.1016/j.biocon.2005.05.002

Fu C, Rao R, Wu J, Chen J, et al. (2003). Effects of density and food availability on growth and cannibalism in basinraising larval salamanders (Hynobius amjiensis). Zool. Res. 24: 186-190.

Gu H and Lau MWN (2004). Hynobius amjiensis. The IUCN Red List of Threatened Species; Version 2015.2. <www. iucnredlist.org>.

Gu H, Mao X, Wang J, Du Z, et al. (1999). Research on population size and dynamics of Hynobius amjiensis. Sichuan Dong $W u$ 18: 104-106.

Guindon S, Dufayard JF, Lefort V, Anisimova M, et al. (2010). New algorithms and methods to estimate maximumlikelihood phylogenies: assessing the performance of PhyML 3.0. Syst. Biol. 59: 307-321. http://dx.doi.org/10.1093/ sysbio/syq010

Hewitt GM (1996). Some genetic consequences of ice ages, and their role in divergence and speciation. Biol. J. Linn. Soc. Lond. 58: 247-276. http://dx.doi.org/10.1111/j.1095-8312.1996.tb01434.x

Ishida S and Taylor DJ (2007). Mature habitats associated with genetic divergence despite strong dispersal ability in an arthropod. BMC Evol. Biol. 7: 52. http://dx.doi.org/10.1186/1471-2148-7-52

Li Y, Wu X, Fang G, Gu C, et al. (2013). Hynobius amjiensis found in Anhui province, China. Chinese J. Zool. 48: 526-528. 
Librado P and Rozas J (2009). DnaSP v5: a software for comprehensive analysis of DNA polymorphism data. Bioinformatics 25: 1451-1452. http://dx.doi.org/10.1093/bioinformatics/btp187

Malyarchuk B, Derenko M, Berman D, Perkova M, et al. (2010). Phylogeography and molecular adaptation of Siberian salamander Salamandrella keyserlingii based on mitochondrial DNA variation. Mol. Phylogenet. Evol. 56: 562-571. http://dx.doi.org/10.1016/j.ympev.2010.04.005

Matsui M, Tominaga A, Hayashi T, Misawa Y, et al. (2007). Phylogenetic relationships and phylogeography of Hynobius tokyoensis (Amphibia: Caudata) using complete sequences of cytochrome $b$ and control region genes of mitochondrial DNA. Mol. Phylogenet. Evol. 44: 204-216. http://dx.doi.org/10.1016/j.ympev.2006.11.031

Monsen KJ and Blouin MS (2003). Genetic structure in a montane ranid frog: restricted gene flow and nuclearmitochondrial discordance. Mol. Ecol. 12: 3275-3286. http://dx.doi.org/10.1046/j.1365-294X.2003.02001.x

Moritz C (1994). Defining ‘evolutionarily significant units' for conservation. Trends Ecol. Evol. (Amst.) 9: 373-375. http://. dx.doi.org/10.1016/0169-5347(94)90057-4

Murphy RW, Fu J, Upton DE, de Lema T, et al. (2000). Genetic variability among endangered Chinese giant salamanders, Andrias davidianus. Mol. Ecol. 9: 1539-1547. http://dx.doi.org/10.1046/j.1365-294x.2000.01036.x

Nei M (1983). Genetic polymorphism and the role of mutation in evolution. In: Nei M, Koehn R (eds) Evolution of genes and proteins. Sinauer Associates, Sunderland, MA, p 165-190.

Pons O and Petit RJ (1996). Measuring and testing genetic differentiation with ordered versus unordered alleles. Genetics 144: $1237-1245$.

Pritchard J and Wen W (2004). Documentation for structure software: version 2. Department of Human Genetics. University of Chicago, Chicago.

Pritchard JK, Stephens M and Donnelly P (2000). Inference of population structure using multilocus genotype data. Genetics 155: 945-959.

Rambaut A, Suchard MA, Xie D and Drummond AJ (2014). Tracer v1. 6. Computer program and documentation distributed by the author, website http://beast. bio. ed. ac. uk/Tracer [accessed 27 July 2014].

Riberon A, Sotiriou E, Miaud C, Andreone F, et al. (2002). Lack of genetic diversity in Salamandra lanzai revealed by cytochrome $b$ gene sequences. Copeia 1: 229-232. http://dx.doi.org/10.1643/0045-8511(2002)002[0229:LOGDIS] 2.0.CO;2

Rogers AR (1995). Genetic evidence for a Pleistocene population explosion. Evolution 1995: 608-615. http://dx.doi. org/10.2307/2410314

Ronquist F, Teslenko M, van der Mark P, Ayres DL, et al. (2012). MrBayes 3.2: efficient Bayesian phylogenetic inference and model choice across a large model space. Syst. Biol. 61: 539-542. http://dx.doi.org/10.1093/sysbio/sys029

Tamura K, Stecher G, Peterson D, Filipski A, et al. (2013). MEGA6: Molecular Evolutionary Genetics Analysis version 6.0. Mol. Biol. Evol. 30: 2725-2729. http://dx.doi.org/10.1093/molbev/mst197

Thompson JD, Gibson TJ, Plewniak F, Jeanmougin F, et al. (1997). The CLUSTAL_X windows interface: flexible strategies for multiple sequence alignment aided by quality analysis tools. Nucleic Acids Res. 25: 4876-4882. http:// dx.doi.org/10.1093/nar/25.24.4876

Vieites DR, Chiari Y, Vences M, Andreone F, et al. (2006). Mitochondrial evidence for distinct phylogeographic units in the endangered Malagasy poison frog Mantella bernhardi. Mol. Ecol. 15: 1617-1625. http://dx.doi.org/10.1111/ j.1365-294X.2006.02874.X

Waits LP, Talbot SL, Ward RH and Shields GF (1998). Mitochondrial DNA phylogeography of the North American brown bear and implications for conservation. Conserv. Biol. 12: 408-417. http://dx.doi.org/10.1046/j.15231739.1998.96351.X

Wang S and Xie Y (2004) China species red list, Vol 1. Beijing, Higher Education Press.

Ye L (2012). Research of the survival status and habitat characteristics of Anji Hynobiid during the breeding season. Master thesis, Zhejiang Normal University.

Zagwijn WH (1992). The beginning of the ice age in Europe and its major subdivisions. Quat. Sci. Rev. 11: 583-591. http:// dx.doi.org/10.1016/0277-3791(92)90015-Z

Zhang H, Yan J, Zhang G and Zhou K (2008). Phylogeography and demographic history of Chinese black-spotted frog populations (Pelophylax nigromaculata): evidence for independent refugia expansion and secondary contact. BMC Evol. Biol. 8: 21. http://dx.doi.org/10.1186/1471-2148-8-21

Zhang M, Rao D, Yang J, Yu G, et al. (2010). Molecular phylogeography and population structure of a mid-elevation montane frog Leptobrachium ailaonicum in a fragmented habitat of southwest China. Mol. Phylogenet. Evol. 54: 47-58. http://dx.doi.org/10.1016/j.ympev.2009.10.019 